\title{
Safety and Efficacy of Dabigatran for Secondary Stroke Prevention in Patients with Nonvalvular Atrial Flbrillation- Multicenter Prospective Registry by Taiwan Stroke Seciety
}

\author{
Ya-Ju Lin ${ }^{1,2}$, Helen.L. Po ${ }^{1,2}$, Ju-Fen Joyce Yeh ${ }^{1}$, Han-Hwa Hu
}

1 MacKay Memorial Hospital, Neurology, Taipei, Taiwan R.O.C..

2 MacKay Medical College, Medicine, New-Taipei, Taiwan R.O.C..

3Taipei Medical University Hospital, Neurology, Taipei, Taiwan R.O.C..

\section{Introduction:}

Stroke in AF patients is usually devastating if not adequately prevented. AF patients with history of stroke/TIA are at higher risk of recurrence. The prescription of adequate oral anticoagulant has been largely changed postNOAC era. Dabigatran, a direct thrombin inhibitor has had been approved as an appropriate alternative oral anticoagulant to prevent stroke or systemic embolism for NVAF patients since Oct 2010 (Jul 2011 in Taiwan) with better safety profile than VKA in clinical trials. Real-world evidence may confirm whether the results of a RCT are observed in everyday clinical practice and can provide additional insight. This multicenter observational prospective cohort study tried to investigate the safety and efficacy of Dabigatran prescribed under routine clinical practice for secondary stroke prevention in NVAF patients in Taiwan.

\section{Methods :}

NVAF Patients, age $\geqq 20 \mathrm{y} / \mathrm{o}$, with history of stroke or TIA, started Dabigatran within 3-months of study initiation or after new index-stroke were enrolled. After patients' consent, we recorded data on their demographics, vital signs, BMI, baseline modified Rankin scale, AF disease information/medical history/risk factors, laboratory tests(including coagulation and lipid profile, liver and renal function) and concomitant medications during baseline, 90-day( \pm 15 days), 180day $( \pm 30$ days) and 360 -day ( \pm 30 days) follow-up visits. Outcome measurement for effectiveness were as follows: stroke/TIA/ICH, myocardial infarction, systemic embolism. Safety outcome measures any bleeding (major/minor), site of bleeding; Gl events and any adverse events. Any cause of death and persistence/adherence of study medication were also recorded.

\section{Results:}

From Dec 2013 till Apr 2017, 653 patients (M/F:399/254) participated, mean age $71.4 \pm 10.4$, mean follow-up 205 days. The median CHA2DS2VASc score was 5; HAS-BLED scale was 3. Two hundred and 4 patients (31.3\%) was newly diagnosed AF. Low dose Dabigatran (110 mg BID) was used in 555(85\%) patients. Standard dose dabigatran (150 mg BID) was prescribed in younger patients (mean age: $64.7 \pm 7.9, \mathrm{p}=0.001$ ) with preserved renal function. Recurrent ischemic stroke was diagnosed in 18 patients/TIA in 2; MI in 5; death in $17(3 \% ; 0.77 \% ; 2.6 \%)(5.45 \% ; 1.36 \% ; 4.6 \%$ per year) (Table 1$)$. Hemorrhagic complications were recorded in 46 patients, 56 events $(8.6 \%$; $12.5 \%$ per year), of which 13 were major bleeding (1.99\%; 3.5\% per year): intracerebral (3), gastrointestinal (7), genitourinary (2), retroperitoneum (1)(table 2 and 3 ). Male, age $\geqq 75 \mathrm{y} / \mathrm{o}$ were risks of bleeding but no difference between low dose and standard dose. Use of statin (nonuser HR : $1.87 ; 95 \% \mathrm{Cl}$ : 1.01 3.47) and $\mathrm{PPI} / \mathrm{H} 2$ blocker have protective effect $(\mathrm{HR}: 0.46 ; 95 \% \mathrm{Cl}$ : $0.22 \sim 0.93) .138(21.1 \%$ ) patients withdrawn prematurely, $37 \%$ due to adverse events.

Table 1. Primary efficacy outcome in TSS dabigatran registry

\begin{tabular}{lllll}
\hline & $\begin{array}{l}90 \text {-day } \\
(\mathbf{n = 6 0 2})\end{array}$ & $\begin{array}{l}\text { 180-day } \\
(\mathbf{n}=\mathbf{4 7 7})\end{array}$ & $\begin{array}{l}\text { 360-day } \\
(\mathbf{n}=350)\end{array}$ & $\begin{array}{l}\text { Total } \\
(\mathbf{n = 6 5 3 )}\end{array}$ \\
\hline Stroke & $5(0.83 \%)$ & $9(1.9 \%)$ & $4(1.1 \%)$ & $18(2.75 \%)$ \\
TIA & $1(0.16 \%)$ & $1(0.2 \%)$ & 0 & $2(0.3 \%)$ \\
CHF & $3(0.5 \%)$ & $2(0.3 \%)$ & 0 & $5(0.77 \%)$ \\
MI & $4(0.83 \%)$ & $1(0.2 \%)$ & 0 & $5(0.77 \%)$ \\
SE & 0 & 0 & 0 & 0 \\
Death & $6(1 \%)$ & $8(1.7 \%)$ & $3(0.86 \%)$ & $17(2.6 \%)$ \\
$\begin{array}{l}\text { sudden CV } \\
\text { death }\end{array}$ & 0 & $2(0.3 \%)$ & $2(0.57 \%)$ & $4(0.61 \%)$ \\
Non CV death & $4(0.66 \%)$ & $4(0.84 \%)$ & 0 & $8(1.2 \%)$ \\
uncertain & $2(0.33 \%)$ & $2(0.3 \%)$ & $1(0.29 \%)$ & $5(0.77 \%)$ \\
\hline
\end{tabular}

Table 2. Safety outcome measurements in TSS dabigatran registry

\begin{tabular}{lllll}
\hline & $\begin{array}{l}90-\text { day } \\
(\mathrm{n}=602)\end{array}$ & $\begin{array}{l}180 \text {-day } \\
(\mathrm{n}=477)\end{array}$ & $\begin{array}{l}360 \text {-day } \\
(\mathrm{n}=350)\end{array}$ & $\begin{array}{l}\text { Total } \\
(\mathrm{n}=653)\end{array}$ \\
\hline Gl events (SAE) & $\begin{array}{l}44(7.3 \%) \\
(3)\end{array}$ & $\begin{array}{l}9(1.9 \%) \\
(0)\end{array}$ & $\begin{array}{l}9(2.6 \%) \\
(3)\end{array}$ & $\begin{array}{l}62(9.5 \%) \\
(5)(0.77 \%)\end{array}$ \\
\hline Abnormal LFT & 1 & 0 & 0 & 1 \\
\hline dyspepsia & 11 & 2 & 1 & 14 \\
\hline $\begin{array}{l}\text { Epigastralgia/ } \\
\text { PUD }\end{array}$ & 19 & 7 & 8 & 34 \\
\hline $\begin{array}{l}\text { Bleeding } \\
\text { (SAE) }\end{array}$ & $31(5.1 \%)$ & $12(2.5 \%)$ & $13(3.7 \%)$ & $56(8.6 \%)$ \\
\hline Others & $(8)$ & $(4)$ & $(6)$ & $(18)(2.8 \%)$ \\
(SAE) & $68(11.3 \%)$ & $37(7.8 \%)$ & $34(9.7 \%)$ & $139(21.3 \%)$ \\
\hline & $(14)$ & $(9)$ & $(12)$ & $(35)(5.4 \%)$ \\
\hline
\end{tabular}

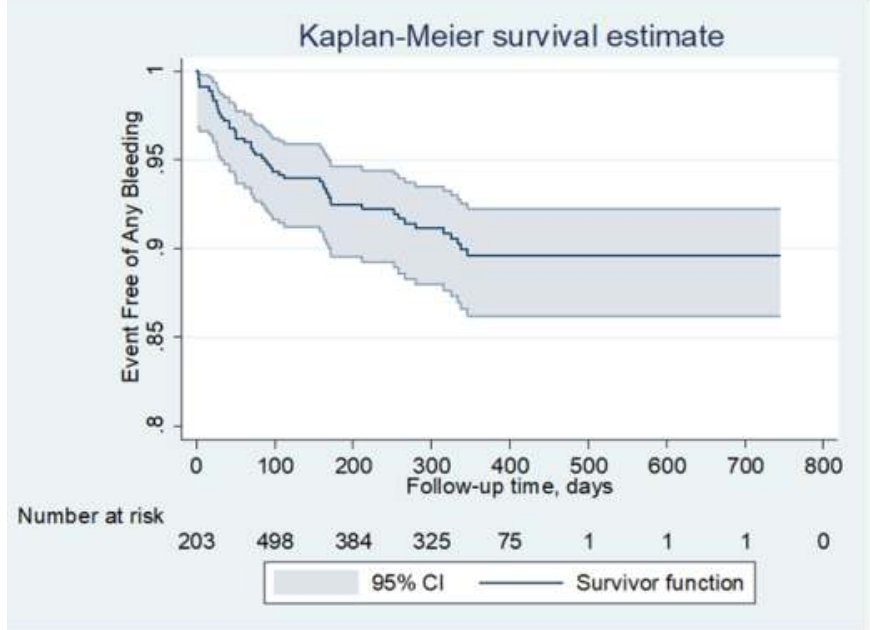

Figure1. The incidence rate of any bleeding events is 10.7 per 100 person-Year

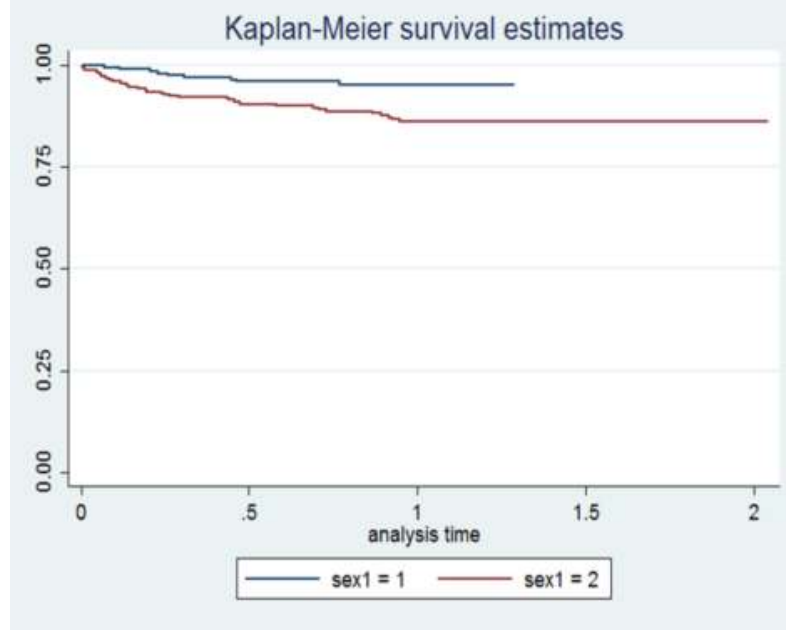

Figure 2. Men have significant higher risk of bleeding (hazard ratio: $4.1,95 \% \mathrm{Cl}: 1.6 \sim 10.5, \mathrm{p}=0.03$ )
Table 3. Comparison of TSS DB registry (all secondary prevention)to RELY trial (20\%) and Taiwan NHI data(39\%)

\begin{tabular}{|c|c|c|c|c|c|}
\hline & $\begin{array}{c}\text { TSS DB registry } \\
2017 \\
\text { Events/pt-year } \\
(n=653)\end{array}$ & $\begin{array}{c}\text { RELY trial } \\
\text { Events/100person- } \\
\text { years }\end{array}$ & $\begin{array}{l}\text { Real world data } \\
\text { from Danish } \\
\text { registry }\end{array}$ & $\begin{array}{c}\text { Taiwan NHI } \\
2010 \sim 13 \\
\text { Dabigatran n=9940 } \\
\text { (per100person- years) }\end{array}$ & $\begin{array}{c}\text { Taiwan NHI } \\
2010 \sim 2013 \text { VKA } \\
n=9913 \\
\text { (per100person- years) }\end{array}$ \\
\hline $\begin{array}{l}\text { Major } \\
\text { bleeding }\end{array}$ & $3.5 \%$ & $\begin{array}{l}\text { DB110: } 2.71 \% \\
\text { DB150: } 3.11 \%\end{array}$ & $\begin{array}{l}\text { DB110: } 2.8 \% \\
\text { DB150: } 2.2 \%\end{array}$ & $1.48 \%$ & $2.47 \%$ \\
\hline $\begin{array}{l}\text { Minor } \\
\text { Bleeding }\end{array}$ & $11.75 \%$ & $\begin{array}{l}\text { DB110:14.62\% } \\
\text { DB150:16.42\% }\end{array}$ & NA & NA & NA \\
\hline Stroke/TIA & $5.45 \%$ & $\begin{array}{l}\text { DB110: } 1.53 \% \\
\text { DB150: } 1.11 \%\end{array}$ & $\begin{array}{l}\text { DB110:2.7\% } \\
\text { DB150:3.5\% }\end{array}$ & $2.96 \%$ & $4.73 \%$ \\
\hline $\mathrm{Ml}$ & $1.36 \%$ & $\begin{array}{l}\text { DB110: } 0.72 \% \\
\text { DB150: } 0.74 \%\end{array}$ & $\begin{array}{l}\text { DB 110:1.0\% } \\
\text { DB150: } 0.9 \%\end{array}$ & $0.66 \%$ & $0.46 \%$ \\
\hline All death & $4.6 \%$ & $\begin{array}{l}\text { DB110: } 3.75 \% \\
\text { DB150: } 3.64 \%\end{array}$ & $\begin{array}{l}\text { DB110:8\% } \\
\text { DB150:3\% }\end{array}$ & $2.70 \%$ & $6.08 \%$ \\
\hline Gl events & $16.9 \%$ & $\begin{array}{l}\text { DB110:11.8\% } \\
\text { DB150: } 11.3 \%\end{array}$ & NA & NA & NA \\
\hline
\end{tabular}

\section{Discussion and Conclusion}

- Our study population is really high risk NVAF patients(CHA2DS2VASc score 5 ), all for secondary stroke prevention with $85 \%$ using low dose dabigatran

- The use of low dose Dabigatran in real-world clinical setting among high-risk NVAF patients in Taiwan was still associated with comparable efficacy and acceptable safety outcomes as in clinical trials

- There's significant lower risk of bleeding in women in this registry which is different from other real-world data

- Our limitation includes small sample size, lack of matched control group and short-duration of follow-up period 